Article

\title{
A Microfluidic Chip Using Phenol Formaldehyde Resin for Uniform-Sized Polycaprolactone and Chitosan Microparticle Generation
}

\section{Yung-Sheng Lin ${ }^{1}$, Chih-Hui Yang ${ }^{2}$, Chin-Tung Wu ${ }^{3}$, Alexandru Mihai Grumezescu ${ }^{4}$, Chih-Yu Wang ${ }^{5}$, Wan-Chen Hsieh ${ }^{2}$, Szu-Yu Chen ${ }^{2}$ and Keng-Shiang Huang ${ }^{6, *}$}

1 Department of Applied Cosmetology and Master Program of Cosmetic Science, Hungkuang University, Taichung 43302, Taiwan; E-Mail: linys@sunrise.hk.edu.tw

2 Department of Biological Science and Technology, I-Shou University, Kaohsiung 82445, Taiwan; E-Mails: chyang@isu.edu.tw (C.-H.Y.); wanjenxie@gmail.com (W.-C.H.); isu9941008a@cloud.isu.edu.tw (S.-Y.C.)

3 Department of Computer Science and Information Engineering, Providence University, Taichung 43301, Taiwan; E-Mail: nxx367@yahoo.com.tw

4 Department of Science and Engineering of Oxidic Materials and Nanomaterials, Faculty of Applied Chemistry and Materials Science, University Politehnica of Bucharest, Bucharest 011061, Romania; E-Mail: grumezescu@yahoo.com

5 Department of Biomedical Engineering, I-Shou University, Kaohsiung 82445, Taiwan; E-Mail: crab@isu.edu.tw

6 The School of Chinese Medicine for Post-Baccalaureate, I-Shou University, Kaohsiung 82445, Taiwan

* Author to whom correspondence should be addressed; E-Mail: huangks@isu.edu.tw; Tel.: +886-7-615-1100 (ext. 7063); Fax: +886-7-615-5150.

Received: 17 April 2013; in revised form: 25 May 2013 / Accepted: 29 May 2013 / Published: 3 June 2013

\begin{abstract}
This study develops a new solvent-compatible microfluidic chip based on phenol formaldehyde resin (PFR). In addition to its solvent-resistant characteristics, this microfluidic platform also features easy fabrication, organization, decomposition for cleaning, and reusability compared with conventional chips. Both solvent-dependent (e.g., polycaprolactone) and nonsolvent-dependent (e.g., chitosan) microparticles were successfully prepared. The size of emulsion droplets could be easily adjusted by tuning the flow rates of the dispersed/continuous phases. After evaporation, polycaprolactone microparticles ranging from 29.3 to $62.7 \mu \mathrm{m}$ and chitosan microparticles ranging from 215.5 to $566.3 \mu \mathrm{m}$ were obtained with a $10 \%$ relative standard deviation in size. The
\end{abstract}


proposed PFR microfluidic platform has the advantages of active control of the particle size with a narrow size distribution as well as a simple and low cost process with a high throughput.

Keywords: microfluidics; emulsion; droplet; microparticles; phenol formaldehyde resin; polycaprolactone; chitosan

\section{Introduction}

Microfluidics is a set of technologies for manipulating nanoliter volumes of fluids in channels with dimensions measured in tenths or even hundreds of micrometers [1]. In the early periods of microfluidic technology in the 1990s, most microfluidic chips were made of silicon and glass [2,3]. Although these materials have good surface qualities and precise properties, there remain some inconveniences in their applications. Silicone or glass/quartz-based products are usually expensive and have low impact strength [4]. Silicone or glass-based fabrication processes are labor intensive and time-consuming, and require costly clean-room facilities and instruments for their photolithography and etching processes, so from a material viewpoint, silicon or glass/quartz-based microfluidic chips have some limitations in practice.

Other rigid materials such as steel or aluminum provide alternative choices for a microfluidic chip substrate [5]. They have advantages such as non-permeable walls, good heat conductivity, robust stability, and durable operation under harsh reaction conditions such as high temperature and/or strong organic solvent systems for certain applications. The limitations of those materials for microfluidic devices are that they are difficult, time-consuming, and costly to fabricate [6].

Poly(dimethylsiloxane) (PDMS) is a soft material and has rapidly become an alternative for microfluidic chips. This material possesses many attractive properties for microfluidics. The major advantages are its easy micromolding fabrication and elastomeric properties. The nontoxic nature and gas permeability also allow its biomedical use. However, its applications suffer from serious swelling and PDMS oligomer leaching in many organic solvents. The limited compatibility of PDMS material with various organic solvents was reported in detail by Whitesides's group [7].

The alternative material for PDMS focuses on the fluorocarbon-based polymer characterized by a high resistance to organic solvents [8-10]. Among them, polytetrafluoroethylene (PTFE) has been widely applied for various microdevices [11-13]. Compared with common commercial polymers, the cost of PTFE is high. This price downside causes several restrictions on its applications.

Introduced in 1909, phenol formaldehyde resin (PFR) is discovered earlier than fluorocarbon polymer. PFR in the form of Bakelite was the earliest commercial synthetic resin. PFR is widely used as a commodity and engineered material in applications such as circuit boards, coatings, adhesives and molded products, including pool balls and laboratory countertops [14]. Recently, biocompatible and green luminescent monodispersed PFR nanoparticles for bioanalytical and biosensing applications have been reported [15]. The PFR material is cheap, solvent-resistant, and easily processed. Furthermore, it is readily available in Taiwan because of Taiwan's important strengths in electronics 
manufacturing and its huge consumer electronics market in the World. However, nowadays few studies have applied the conventional PFR material for microfluidic chips.

Polycaprolactone (PCL) and chitosan microspheres have gained a great deal of attention for biomedical applications. These microspheres can be used as a means of enhancing drug delivery efficiency, biomedical diagnostics and target manipulation. PCL is non-toxic in nature and found to be cyto-compatible with a several body tissues, which makes it a perfect material for tissue engineering. PCL has flexible mechanical properties that are suitable for paramedical applications, wound dressings, and dentistry [16,17]. This substantiates the functionality of the polymer in pharmaceutical dosage form design and development. Chitosan, a natural biodegradable polymer, is of great interest in biomedical research due to its excellent properties including bioavailability, nontoxicity, high charge density, and mucoadhesivity, which creates an immense potential for various biomedical and pharmaceutical applications such as drug delivery, tissue engineering, cosmetics, and food and nutrition products [18-21]. Hydrophobic PCL and hydrophilic chitosan can be considered representative of solvent- and nonsolvent-dependent polymers, respectively. We use these two representative polymers to demonstrate the compatibility of the PFR chip with the organic solvent and water.

Based on our previous microfluidic platforms for the generation of various polymer particles [22-31], this study provides a new solvent-compatible microfluidic chip based on PFR to prepare uniform microparticles. This is the first report using the PFR microfluidic chip to generate both hydrophobic and hydrophilic material microparticles. The solvent-dependent polycaprolactone and nonsolventdependent chitosan were demonstrated to be applicable on the same platform. The proposed PFR microfluidic platform provides great promise for applications in particle generation.

\section{Results and Discussion}

Conventional microfluidic chips are difficult to clean properly because of the bonded sealing design [32-35]. To overcome this drawback, the proposed PFR microfluidic chip is designed to be disassembled for microchannel cleaning. Three layers connected by twenty M4 screws could be easily taken apart for further cleaning after each experiment.

Generally, phenol formaldehyde resin can dissolve in many organic solvents. The prepared PFR microfluidic chip thus has some limitations for a long contact with organic solvents. However, there were no problems in the synthesis of polymer microparticles using the PFR microfluidic chip for $24 \mathrm{~h}$ with chloroform in this study, as shown in Figure 1.

Figure 1. Appearance of the cross-junction microchannel in the PFR chip before (A) and after (B) contact with chloroform for $24 \mathrm{~h}$.
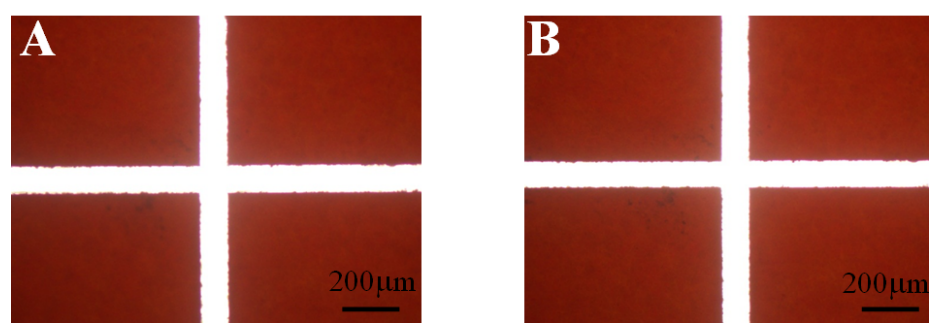
Figure 2 shows PCL microparticles collected under the condition of a $0.3 \mathrm{~mL} / \mathrm{min}$ continuous flow rate and a $0.2 \mathrm{~mL} / \mathrm{h}$ dispersed flow rate. The scanning electron microscope (SEM) picture reveals that PCL microparticles were consistent in morphology, and exhibited excellent size uniformity (Figure 2A). The average diameter of the PCL microparticles was $46.1 \pm 3.1 \mu \mathrm{m}$. The zoom-in image (Figure 2B) shows that of the PCL microparticles had smooth surface and good sphericity.

Figure 2. Pictures of PCL microparticles in scanning electron microscope. (A) magnification $400 \times$ and (B) magnification 7,000×.

(A)

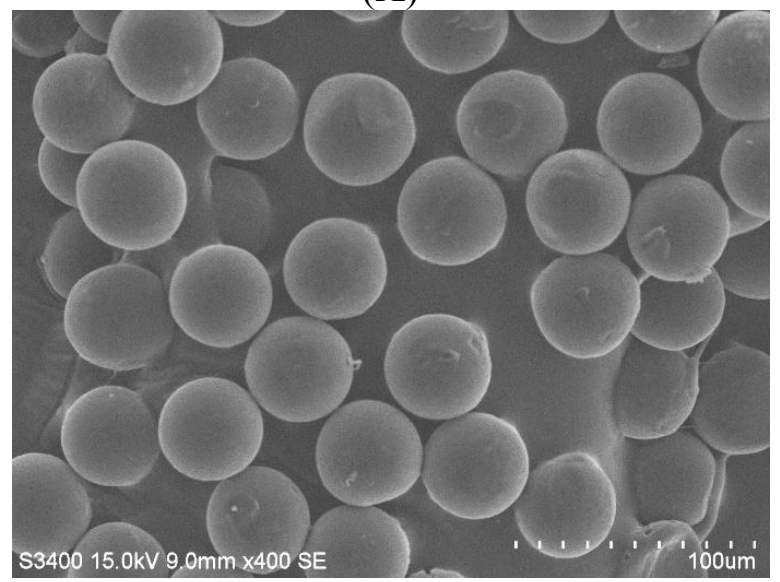

(B)

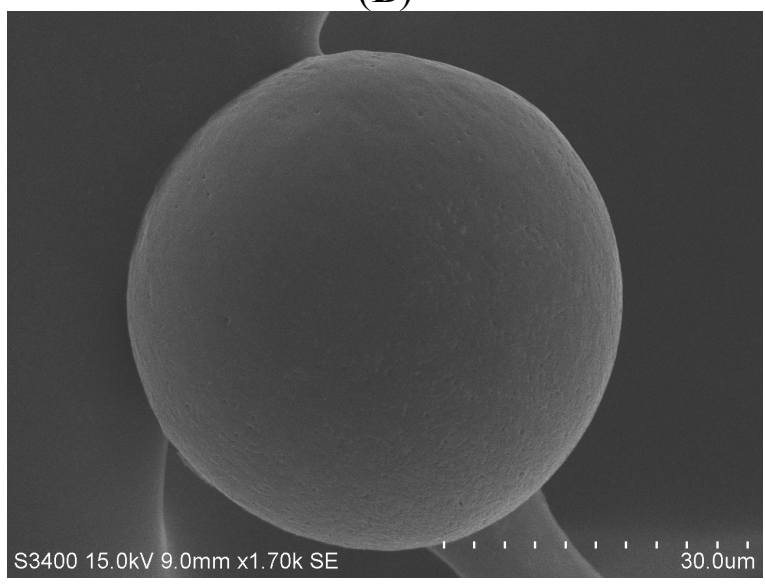

Figure 3 displays chitosan microparticles collected under the condition of a $0.5 \mathrm{~mL} / \mathrm{minute}$ continuous flow rate and a $0.04 \mathrm{~mL} /$ hour dispersed flow rate. Compared with PCL microparticles, chitosan microparticles were less consistent in both morphology and size due to their less rigid properties (Figure 3A). In spite of the differences in chitosan microparticle sizes, the size of microparticles could still be controlled within about a $10 \%$ relative standard deviation (R.S.D., defined as the ratio of standard deviation to average). The average diameter of the chitosan microparticles was $215.5 \pm 8.9 \mu \mathrm{m}$. The chitosan microparticle had some sunken regions and a wrinkled surface as shown in Figure 3B.

Figure 3. Pictures of chitosan microparticles in scanning electron microscope. (A) magnification $100 \times$ and (B) magnification $400 \times$.

(A)

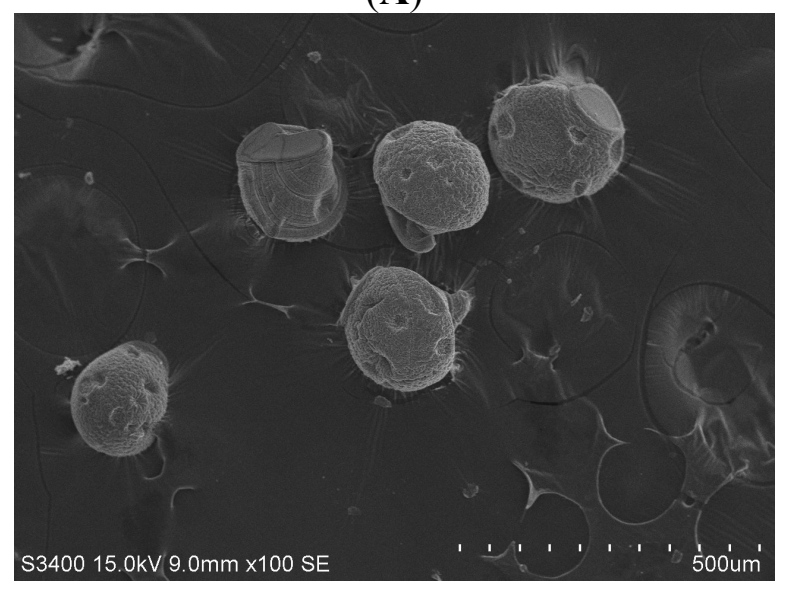

(B)

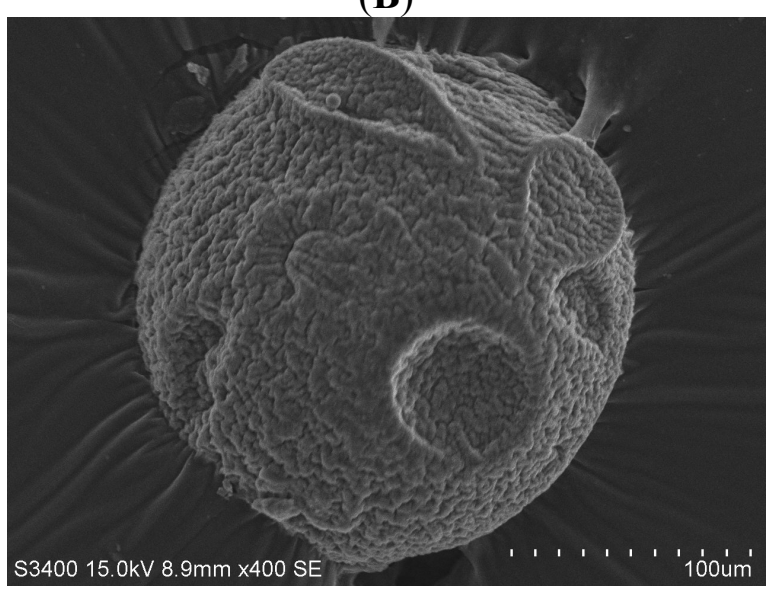


Figures 4 and 5 show the relationships between droplet/microparticle sizes and continuous/dispersed flow rates for PCL and chitosan, respectively. The small relative standard deviation in size of particles including emulsion droplets and dry solid microparticles indicates the good uniformity of particles obtained in this microfluidic platform. This uniformity corresponds to the results of monodispersed microparticles in Figures 2A and 3A.

Figure 4. Droplet size (A), microparticle size (B), and shrinking ratio (C) of polycaprolactone in different continuous/dispersed flow rates.

(A)

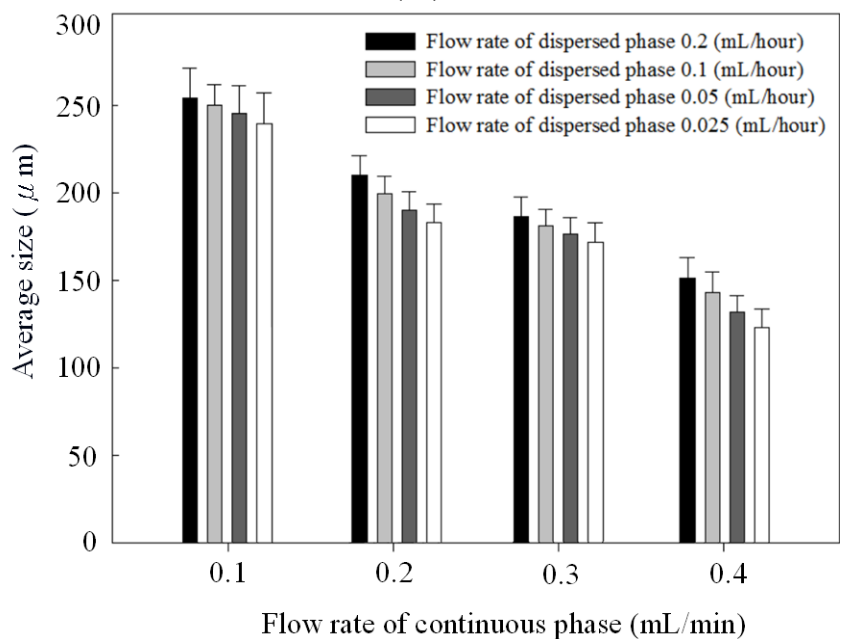

(B)

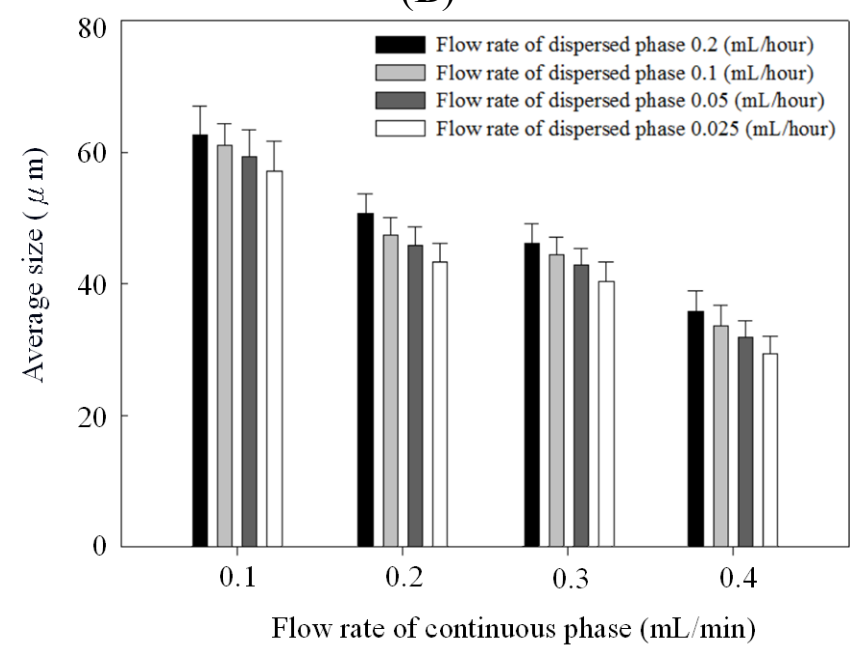

(C)

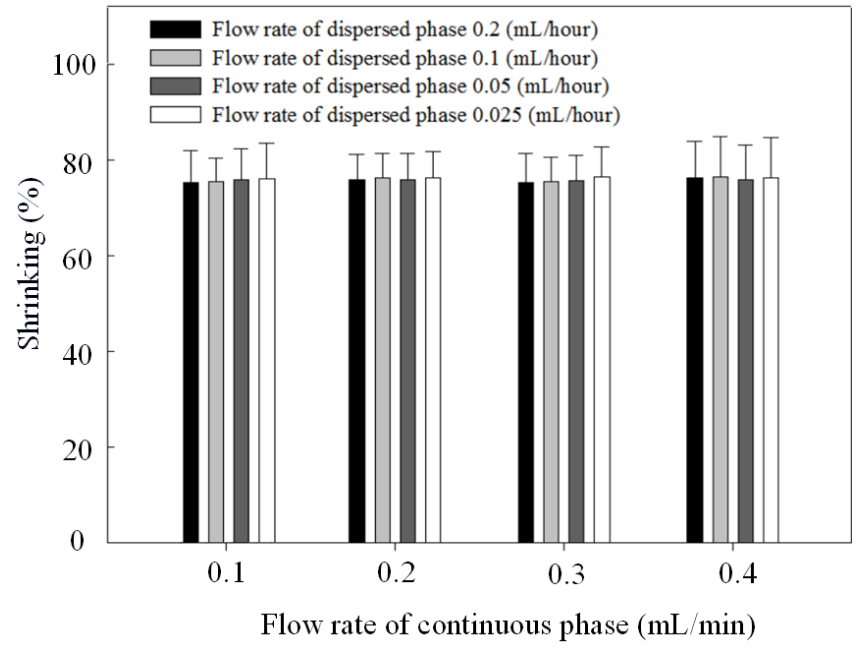

At the same concentration of polymer solution $(1 \% \mathrm{wt} / \mathrm{v})$, both emulsion droplets and dry solid microparticles of PCL were smaller than chitosan. The emulsion droplet size with different materials (polycaprolactone and chitosan) varied significantly, resulting from the different competition between viscous stresses and capillary stresses between the two phases. Furthermore, the shrinking ratio (from a droplet to a dry microparticle) of PCL was higher than for chitosan, probably due to a more dense structure in the PCL microparticles. In the solvent evaporation process, polycaprolactone molecules rearranged and packed to condense the emulsion droplet to be the dry microparticle. There was a high shrinkage ratio in this solidification process. However, chitosan molecules underwent an extra 
crosslinking to form a gel in the emulsion droplet. Compared with polycaprolactone, the crosslinked chitosan molecules had lower mobility for rearrangement and packing in the evaporation process, resulting in a low shrinkage ratio. Therefore, polycaprolactone droplets have a higher shrinkage ratio than chitosan.

Figure 5. Droplet size (A), microparticle size (B), and shrinking ratio (C) of chitosan in different continuous/dispersed flow rates.

(A)

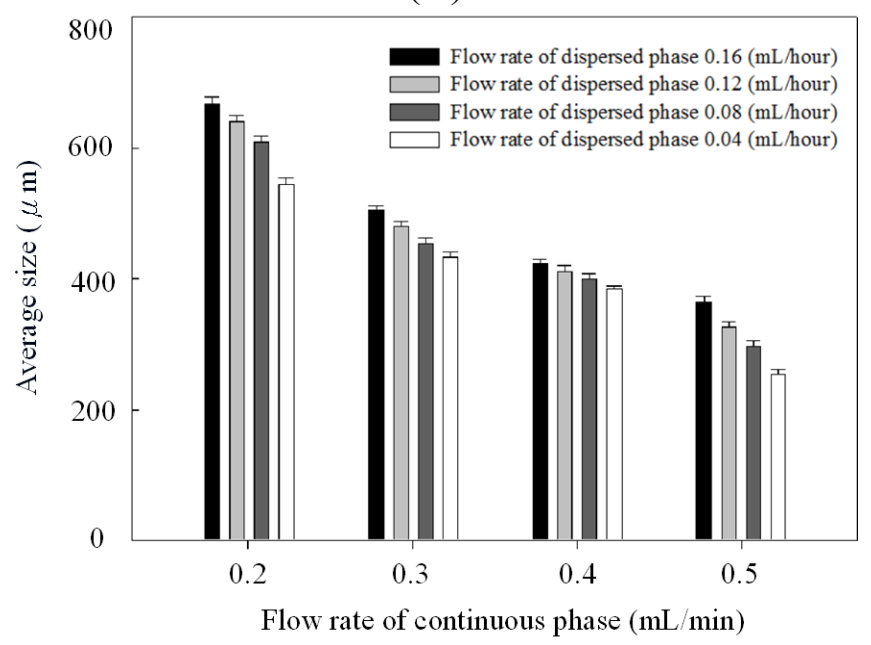

(B)

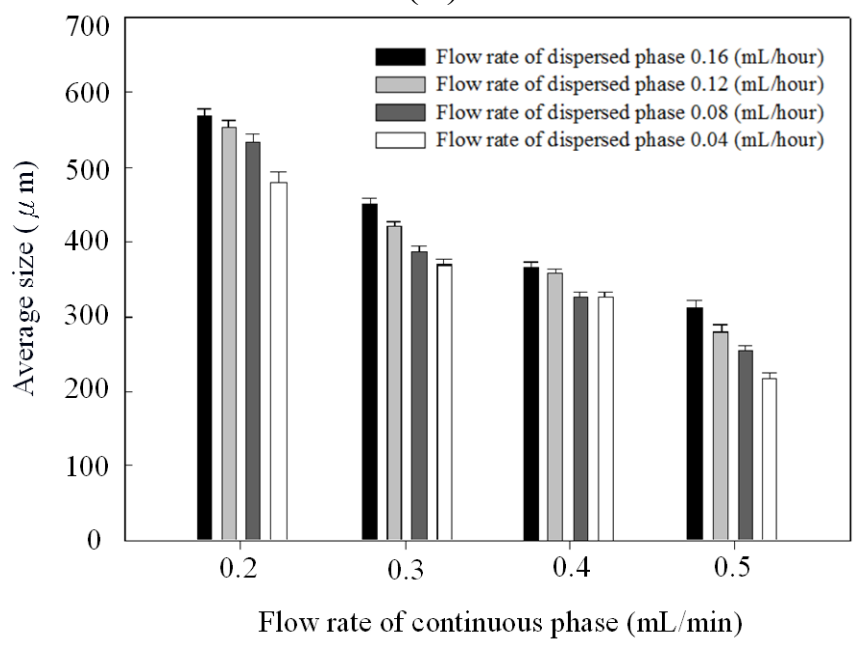

(C)

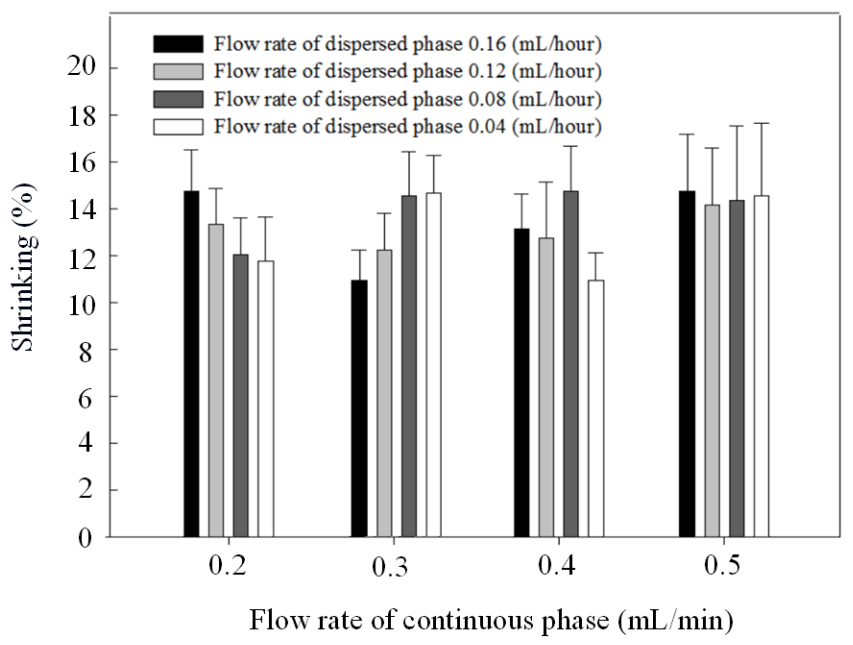

Figures $4 \mathrm{~B}$ and $5 \mathrm{~B}$ indicate that particles decreased with the continuous phase flow rate but increased with the dispersed phase flow rate. Effects of continuous/dispersed flow rates on the particle size agree with previous reports [22-31]. A higher continuous flow rate provided a greater shear force to accelerate the detachment of emulsion droplets from the dispersed phase flow. The shorter droplet formation time of the dispersed phase flow resulted in a smaller emulsion droplet in size. The $0.1-0.4 \mathrm{~mL} / \mathrm{min}$ flow rate of PVA solution in PCL system and $0.2-0.5 \mathrm{~mL} / \mathrm{min}$ of sunflower seed oil in chitosan system show the smaller particle size at a higher continuous flow rate.

On the contrary, a higher dispersed flow rate increased the emulsion droplet size. Under the same droplet formation mechanism, the droplet volume increased with the dispersed flow rate due to the accumulated volume in a droplet. Consequently the size of particles is larger in a higher dispersed flow 
rate. The flow rate of $0.025 \sim 0.2 \mathrm{~mL} / \mathrm{h}$ for PCL solution and $0.04-0.16 \mathrm{~mL} / \mathrm{h}$ for chitosan solution show the larger particle size at a higher dispersed flow rate.

Different from droplet formation at the cross-junction in the microchannel [22-31], we used the jetting flow with a long neck to adjust the shear force breaking up the immiscible polymer solution into big emulsion droplets. Although the neck was long, it was still stable enough for regular emulsion droplet formation. This result agreed with the classic literature concerning liquid/liquid jet dispersion [36-41]. As shown in the SEM pictures, polycaprolactone and chitosan microparticles obtained from the jetting flow were uniform in both morphology and size.

\section{Experimental}

\subsection{Materials}

Poly(vinyl alcohol) (PVA, 88\%-89\% hydrolyzed), polycaprolactone (PCL), and chitosan were purchased from Sigma (Sigma Chemical Co., St. Louis, MO, USA). Sunflower seed oil was obtained from Uni-President Enterprises Corp., Tainan, Taiwan. Distilled water was filtered by a $0.22 \mathrm{~nm}$ filter (Millipore Inc., Clifton, NJ, USA) before use. All reagents were used as purchased without any further purification.

\subsection{Construction of the Microfluidic Platform}

The proposed microfluidic chip was laid out on a conventional PFR substrate (length/width/depth: $270 \mathrm{~mm} / 210 \mathrm{~mm} / 1 \mathrm{~mm}$ ). The PFR microfluidic chip was fabricated using an engraving micromachining process by an engraving machine (Twinsoft, Taipei, Taiwan). The microfluidic device (Figure 6A) consists of three layers which are from top to bottom: the cover layer (containing three inlets and twenty screw orifices for binding), the main layer (containing main microfluidic channel and screw orifices) and the bottom layer (containing one outlet and twenty screw orifices for binding), respectively. These three layers were integrated by twenty M4 screws ( $0.5 \mathrm{~mm}$ pitch, $4 \mathrm{~mm}$ diameter) to be the microfluidic device for preparing microparticles. Figure $6 \mathrm{~B}$ shows the geometric dimension of the microchannel with a $100 \mu \mathrm{m}$ wide cross-junction. The downstream of the cross-junction was designed to be gradually broadened to $2000 \mu \mathrm{m}$ in width for slowing down the flow and enhancing observation of microdroplets. Figure $6 \mathrm{C}$ displays a photograph of the PFR microfluidic chip.

\subsection{Experimental Procedure}

Polycaprolactone and chitosan droplets were obtained by the emulsion process. The pair of dispersed and continuous phases for these two materials were polycaprolactone solution $(1 \% \mathrm{wt} / \mathrm{v}$ in chloroform) and PVA ( $1 \% \mathrm{wt} / \mathrm{v}$ in distilled water), and chitosan solution ( $1 \% \mathrm{wt} / \mathrm{v}$ in $2 \%$ acetic acid) and sunflower seed oil, respectively. Two sets of independently controlled syringe pumps (Kd Scientific KDS101, Holliston, MA, USA) were used to separately inject dispersed and continuous phases into the microfluidic chip together. The dispersed phase was injected into the central channel, while the continuous phase was injected from the two side channels (Figure 7) hydrodynamically focusing a stream of polymers solution enabled the construction of polymer emulsions along the microchannel axis. The shear force at the downstream expansion site of cross-junction stretched 
dispersed phase into a droplet periodically as shown in the picture of Figure 7. These collected droplets further made process of evaporation and solidification in a $50 \mathrm{~mL}$ beaker at $37{ }^{\circ} \mathrm{C}$ for $24 \mathrm{~h}$. All droplets were lyophilized for another $24 \mathrm{~h}$ to complete liquid removal to become dry solid microparticles.

Figure 6. The proposed phenol formaldehyde resin-based microfluidic device: (A) The chip device in expanded view. (B) Geometry of the microfluidic channel. (C) A photograph of the microfluidic chip.

A
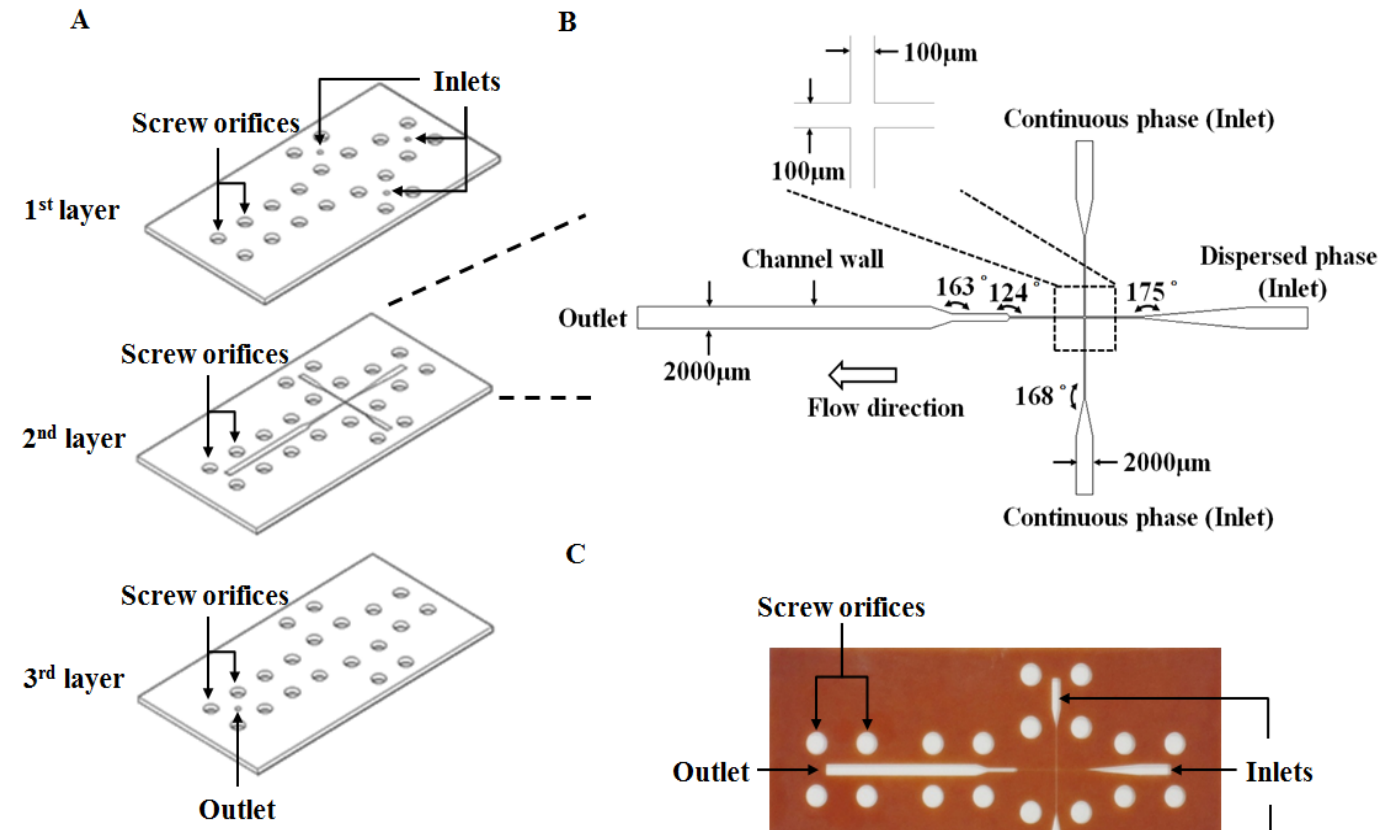

B

C

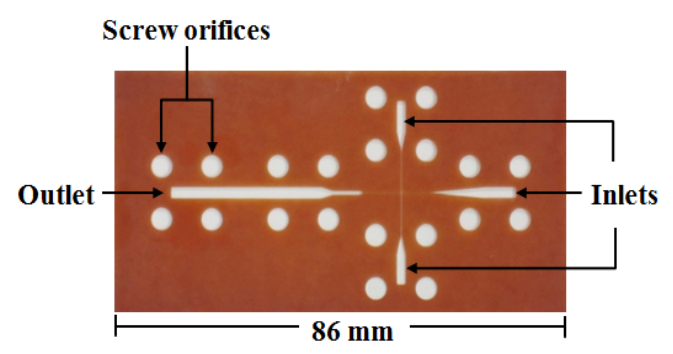

Figure 7. Schematic drawing of droplet formation in the cross-junction microchannel. The flow-focusing system generates uniform self-assembling droplets at the expansion site of the main channel.

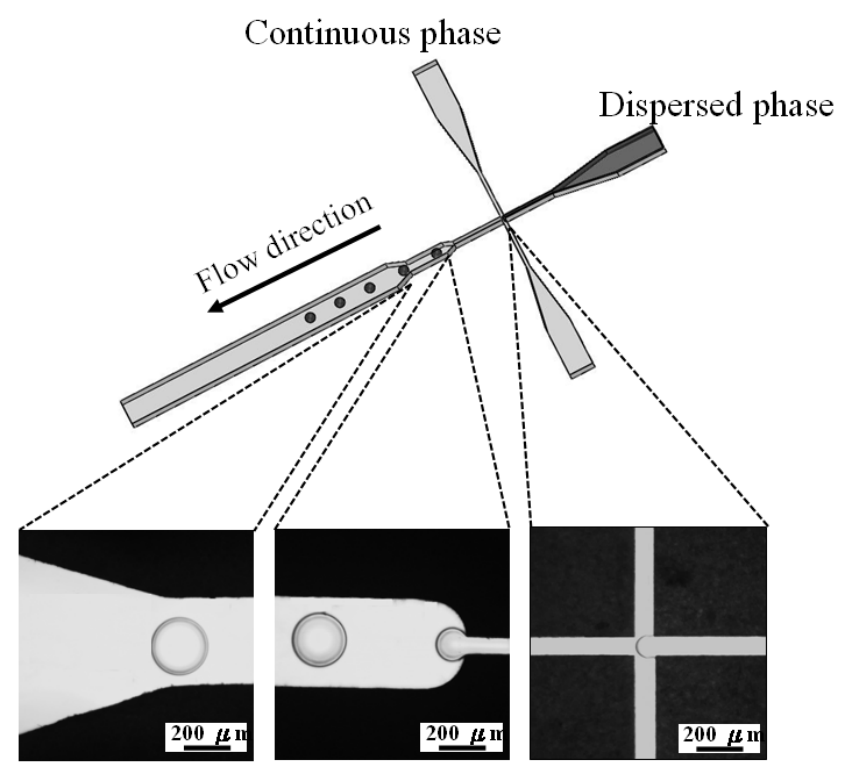


An optical microscope (TE2000U, Nikon, Lewisville, TX, USA) and scanning electron microscope (S-2700, Hitachi, Tokyo, Japan) were used to observe emulsion droplets and dry solid microparticles, respectively. To ensure statistical representation, one hundred particles including emulsion droplets and dry solid microparticles were counted to calculate the average particle size. The diameter of the particles was expressed as mean \pm standard deviation.

\section{Conclusions}

This study developed a new microfluidic platform for uniform microparticle preparation. The phenol formaldehyde resin-based microfluidic chip is solvent-resistant and can be disassembled for cleaning to be reused. Prepared polycaprolactone and chitosan microparticles were uniform in size, and their sizes could be adjusted by changing the flow rates of the dispersed/continuous phases. Polycaprolactone microparticles had a higher shrinkage ratio than chitosan ones during the evaporation process. The proposed microfluidic platform is low cost, easy to organize and offers high throughput for uniform microparticle generation.

\section{Acknowledgments}

This work was financially supported by a grant from the National Science Council of Taiwan, R.O.C.

\section{Conflict of Interest}

The authors declare no conflict of interest.

\section{References}

1. Whitesides, G.M. The origins and the future of microfluidics. Nature 2006, 442, 368-373.

2. Ouellette, J. A new wave of microfluidic devices. Ind. Phys. 2003, 9, 14-17.

3. Quake, S.R.; Scherer, A. From micro- to nanofabrication with soft materials. Science 2000, 290, $1536-1540$.

4. Lee, K.; Kim, C.; Shin, K.S.; Lee, J.W.; Ju, B.K.; Kim, T.S.; Lee, S.K.; Kang, J.Y. Fabrication of round channels using the surface tension of PDMS and its application to a $3 \mathrm{D}$ serpentine mixer. J. Micromech. Microeng. 2007, 17, 1533-1541.

5. Lin, Y.S.; Yang, C.H.; Wang, C.Y.; Chang, F.R.; Huang, K.S.; Hsieh, W.C. An aluminum microfluidic chip fabrication using a convenient micromilling process for fluorescent poly(DL-lactide-co-glycolide) microparticle generation. Sensors 2012, 12, 1455-1467.

6. Becker, H.; Locascio, L.E. Review: Polymer microfluidic devices. Talanta 2002, 56, 267-287.

7. Lee, J.N.; Park, C.; Whitesides, G.M. Solvent compatibility of poly(dimethylsiloxane)- based microfluidic devices. Anal. Chem. 2003, 75, 6544-6554.

8. Rolland, J.P.; Van Dam, R.M.; Schorzman, D.A.; Quake, S.R.; DeSimone, J.M. Solvent-resistant photocurable liquid fluoropolymers for microfluidic device fabrication. J. Am. Chem. Soc. 2004, 126, 2322-2323.

9. De Marco, C.; Girardo, S.; Mele, E.; Cingolani, R.; Pisignano, D. Ultraviolet-based bonding for perfluoropolyether low aspect-ratio microchannels and hybrid devices. Lab Chip 2008, 8, 1394-1397. 
10. Renckens, T.J.; Janeliunas, D.; van Vliet, H.; van Esch, J.H.; Mul, G.; Kreutzer, M.T. Micromolding of solvent resistant microfluidic devices. Lab Chip 2011, 11, 2035-2038.

11. Rahman, P.K.; Pasirayi, G.; Auger, V.; Ali, Z. Development of a simple and low cost microbioreactor for high-throughput bioprocessing. Biotechnol. Lett. 2009, 31, 209-214.

12. Castell, O.K.; Allender, C.J.; Barrow, D.A. Liquid-liquid phase separation: Characterisation of a novel device capable of separating particle carrying multiphase flows. Lab Chip 2009, 9, 388-396.

13. Rowe, D.J.; Porch, A.; Barrow, D.A.; Allender, C.J. Microfluidic device for compositional analysis of solvent systems at microwave frequencies. Sens. Actuators B Chem. 2012, 169, 213-221.

14. Raqueza, J.M.; Deléglisea, M.; Lacrampea, M.F.; Krawczaka, P. Thermosetting (bio)materials derived from renewable resources: A critical review. Prog. Polym. Sci. 2010, 35, 487-509.

15. Yang, P.; Zhao, Y.; Lu, Y.; Xu, Q.Z.; Xu, X.W.; Dong, L.; Yu, S.H. Phenol formaldehyde resin nanoparticles loaded with CdTe quantum dots: A fluorescence resonance energy transfer probe for optical visual detection of copper(II) ions. ACS Nano. 2011, 5, 2147-2154.

16. Woodruff, M.A.; Hutmacher, D.W. The return of a forgotten polymer-polycaprolactone in the 21st century. Prog. Polym. Sci. 2010, 35, 1217-1256.

17. Estelle, J.M.; Vidaurre, A.; Duenas, J.M.M.; Cortazar, I.C. Physical characterization of polycaprolactone scaffolds. J. Mater. Sci. Mater. Med. 2008, 19, 189-195.

18. Jayakumar, R.; Prabaharan, M.; Sudheesh Kumar, P. T.; Nair, S. V.; Tamura, H. Biomaterials based on chitin and chitosan in wound dressing applications. Biotechnol. Adv. 2011, 29, 322-337.

19. Bruno, K. Using drug-excipient interactions for siRNA delivery. Adv. Drug Deliv. Rev. 2011, 63, 1210-1226.

20. Merkel, O. M.; Zheng, M.; Debus, H.; Kissel, T. Pulmonary gene delivery using polymeric nonviral vectors. Bioconjug. Chem. 2012, 23, 3-20.

21. Kim, H.; Cooke, M.J.; Shoichet, M.S. Creating permissive microenvironments for stem cell transplantation into the central nervous system. Trends Biotechnol. 2012, 30, 55-63.

22. Yang, C.H.; Lin, Y.S.; Huang, K.S.; Huang, Y.C.; Wang, E.C.; Jhong, J.Y.; Kuo, C.Y. Microfluidic emulsification and sorting assisted preparation of monodisperse chitosan microparticles. Lab Chip 2009, 9, 145-150.

23. Yang, C.H.; Huang, K.S.; Lin, Y.S.; Lu, K.; Tzeng, C.C.; Wang, E.C.; Lin, C.H.; Hsu, W.Y.; Chang, J.Y. Microfluidic assisted synthesis of multi-functional polycaprolactone microspheres: Incorporation of CdTe quantum Dots, $\mathrm{Fe}_{3} \mathrm{O}_{4}$ superparamagnetic nanoparticles, and tamoxifen. Lab Chip 2009, 9, 961-965.

24. Huang, K.S.; Lu, K.; Yeh, C.S.; Chung, S.R.; Lin, C.H.; Yang, C.H.; Dong, Y.S. Microfluidic controlling monodisperse microdroplet for 5-fluorouracil loaded genipin-gelatin microcapsules. J. Contr. Release 2009, 127, 15-19.

25. Huang, K.S.; Yang, C.H.; Lin, Y.S.; Wang, C.Y.; Lu, K.; Chang, Y.F.; Wang, Y.L. Electrostatic droplets assisted synthesis of alginate microcapsules. Drug Deliv. Transl. Res. 2011, 1, 289-298.

26. Huang, K.S.; Lin, Y.S.; Yang, C.H.; Tsai, C.W.; Hsu, M.Y. In situ synthesis of twin monodispersed alginate microparticles. Soft Matter 2011, 7, 6713-6718.

27. Lin, Y.S.; Yang, C.H.; Lu, K.; Huang, K.S.; Zheng, Y.Z. Synthesis of agar microparticles using temperature-controlled microfluidic devices for Cordyceps Militaris cultivation. Electrophoresis 2011, 32, 3157-3163. 
28. Yang, C.H.; Huang, K.S.; Grumezescu, A.M.; Wang, C.Y.; Tzeng, S.C.; Chen, S.Y.; Lin, Y.H.; Lin, Y.S. Synthesis of uniform PLA and PLGA microspheres using a microfluidic chip for comparison. Electrophoresis 2013, in press.

29. Yang, C.H.; Wang, C.Y.; Huang, K.S.; Yeh, C.S.; Wang, A.H.; Wang, W.T.; Lin, M.Y. Facile synthesis of radial-like macroporous superparamagnetic chitosan spheres with in-situ co-precipitation and gelation of ferro-gels. PLoS One 2012, 7, e49329.

30. Yang, C.H.; Huang, K.S.; Wang, C.Y.; Hsu, Y.Y.; Chang, F.R.; Lin, Y.S. Microfluidic-assisted synthesis of hemispherical and discoidal chitosan microparticles at an oil/water interface. Electrophoresis 2012, 33, 3173-3180.

31. Lin, Y.S.; Yang, C.H.; Hsu, Y.Y.; Hsieh, C.L. Microfluidic synthesis of tail-shaped alginate microparticles using slow sedimentation. Electrophoresis 2013, 34, 425-431.

32. Lee, M.; Lee, J.; Rhee, H.; Choo, J.; Chai, Y.G.; Lee, E.K. Applicability of laser-induced Raman microscopy for in situ monitoring of imine formation in a glass microfluidic chip. J. Raman Spectrosc. 2003, 34, 737-742.

33. Jia, Z.J.; Fang, Q.; Fang, Z.L. Bonding of glass microfluidic chips at room temperatures. Anal. Chem. 2004, 76, 5597-5602.

34. Qu, B.Y.; Wu, Z.Y.; Fang, F.; Bai, Z.M.; Yang, D.Z.; Xu, S.K. A glass microfluidic chip for continuous blood cell sorting by a magnetic gradient without labeling. Anal. Bioanal. Chem. 2008, 392, 1317-1324.

35. Coltro, W.K.; de Jesus, D.P.; da Silva, J.A.; do Lago, C.L.; Carrilho, E. Toner and paper-based fabrication techniques for microfluidic applications. Electrophoresis 2010, 31, 2487-2498.

36. Scheele, G.F.; Meister, B.J. Drop formation at low velocities in liquid-liquid systems: Part I. Prediction of drop volume. AIChE J. 1968, 14, 9-15.

37. Izard, J.A. Prediction of drop volumes in liquid-liduid systems. AIChE J. 1972, 18, 634-638.

38. Kagan, S.Z.; Kovalev, N.; Zakharychev, A.P. Drop size with drop-type flow conditions out of nozzles in liquid/liquid systems. Theor. Found. Chem. Eng. 1973, 7, 514-518.

39. Clift, R.; Grace, J.R.; Weber, M.E. Formation and breakup of fluid particles. In Bubbles, Drops, and Particles; Academic Press: New York, NY, USA, 1978; pp. 321-351.

40. Kumar, A.; Hartland, S. Correlation for drop size in liquid/liquid spray columns. Chem. Eng. Commun. 1984, 31, 193-207.

41. Zhang, X. Dynamics of drop formation in viscous flows. Chem. Eng. Sci. 1999, 54, 1759-1774.

Sample Availability: Not available.

(C) 2013 by the authors; licensee MDPI, Basel, Switzerland. This article is an open access article distributed under the terms and conditions of the Creative Commons Attribution license (http://creativecommons.org/licenses/by/3.0/). 